Revista de Matemática: Teoría y Aplicaciones 1999 6(1) : 67-84

CIMPA - UCR - CCSS ISSN: 1409-2433

\title{
ON A VARIATIONAL PRINCIPLE FOR SHAPE OPTIMIZATION AND ELLIPTIC FREE BOUNDARY PROBLEMS
}

\author{
Raúl B. González De PaZ*
}

Received: November 11, 1998

\begin{abstract}
A variational principle for several free boundary value problems using a relaxation approach is presented. The relaxed Energy functional is concave and it is defined on a convex set, so that the minimizing points are characteristic functions of sets. As a consequence of the first order optimality conditions, it is shown that the corresponding sets are domains bounded by free boundaries, so that the equivalence of the solution of the relaxed problem with the solution of several free boundary value problem is proved.
\end{abstract}

Keywords: Calculus of variations, optimization, free boundary problems.

\section{Resumen}

Se presenta un principio variacional para varios problemas de valores en fronteras libres usando un enfoque de relajamiento. El funcional de Energía relajado es cóncavo y está definido en un conjunto convexo, de tal forma que los puntos que minimizan son funciones características de conjuntos. Como consecuencia de las condiciones de optimalidad de primer orden, se muestra que los conjuntos correspondientes son dominios acotados por fronteras libres, de manera que se prueba la equivalencia de la solución del problema relajado con la solución de varios problemas de valores en fronteras libres.

Palabras-clave: Cálculo de variaciones, optimización, problema de frontera libre.

AMS Subject Classification: 35J35, 49A22, 49B22.

* Departamento de Matemáticas, Universidad del Valle de Guatemala, Apartado Postal 82, 01901 Guatemala, Guatemala 


\section{Introduction}

During the last decade there has been an active research in two fields: shape optimization and free boundary value problems. With the help of variational principles, it seems clear now that at least for some cases, there are common results for both subjects. Once an "energy" functional $\Omega \rightarrow E(\Omega)$ depending on the shape of the domain $\Omega$ is defined, if there exists a domain $\Omega *$ which minimizes the energy, it can be shown under suitable regularity assumptions, that the boundary of the optimal domain can be interpreted as the solution of a free bounday problem as a consequence of first-order optimality conditions. One of the main contributions of the variational analysis during the last years has been the well-known paper by Alt-Cafarelli [1], but other contributions have appeared recently (see for instance the article by J.P. Zolesio [36]). The work developed by the shape optimization community has been mainly classified in two directions: one is the work done on shape sensitivity analysis, where gradients of the domain functionals have been calculated (see works by Delfour [7], Murat-Simon [23], Pironneau [25], Simon [27], Fujii [10], Sokolowski-Zolesio [28], Zolesio [34]). On the other side, the research on relaxation methods allows to work within more general functional space frameworks. Those have been studied, among others, by Kohn-Strang [21] , Buttazo-Dal Maso [3], Kohn [20], Murat-Tartar [24], Gonzales de Paz [13], [15]. This approach has been used mainly to obtain existence results, but generally, what we obtain are some kind of "generalized domains". Additional regularity conditions are imposed in order to obtain "classical domains" (cf. for example D. Chenais [6]). It seems clear now that, so far we deal with some kind of energy functionals, (which is not always the case with shape-optimization problems) and in case the relaxation factor plays the role of a control-coefficient for other terms than the quadratic gradient-term, then a homogeneization phenomenon does not occur, but instead a free boundary value problem is solved. An important fact that it has not been remarked elsewhere, as far as the author knows, is that the correponding energy functionals are concave related to the relaxation term (control coefficient). The concavity structure provides the "good" structure in order to obtain existence results and differentiability, as there is no need to restrict ourselves to sets of characteristic funtions; they appear as a consequence of the concavity. This allows one to use more general function sets, and apply well-known compacity results for topological vector spaces. Similar problems have been treated within the control-theory framework by R. Tahraoui [29].

Our goal in this paper is to present a relaxation approach for the study of free boundary value problems and free interface boundary problems which seems to describe a general variational principle. Generally, a condition of the Dirichtlet-type is given on the free boundary and another condition concerning the normal derivative of the function is prescribed, (a Neumann or Bernoulli condition). For the case of interface boundaries, the additional condition on the normal derivative takes the form of a "transmission" condition. By means of this approach, introduced by González de Paz [13], a constructive proof for the existence of optimal domains is presented, in the sense that a concave functional is given whose minimizing elements describe the domain or subdomains bounded by the free boundary. Under certain restrictions, the homogeneization process does not take place and we obtain "classical" domains. As the functional is differentiable, a further study of 
the necessary conditions of optimality using the techniques of shape derivative calculation developed by J.P. Zolesio [36] are used for the study of the boundary conditions on the free interface boundary.

We shall deal with two main applications: firstly we show how this relaxation approach can be used for the study of steady vortex flow problems, this is a rather classic free boundary value problems, but it allows to present the main features of our approach. Secondly, we study the shape optimization of electrostatic condensators, in the sense that, given one of the boundaries of the condensator, we look for the other one in order to maximize the corresponding capacity. This kind of problems have found some applications in heat-conduction, electrochemistry, fluid mechanics and plasma physics. In order to apply the relaxation approach, we have to use some kind of penalization or regularization, depending on the case we are dealing. In a second stage, we study the case where the normal derivative of the solution of the partial differential equation is related to the curvature of the free boundary, we shall call this conditions of the non linear Bernoulli-type. This can be handled within our framework by adding a new term to the functional. We show that the main results remain valid, and in fact, the new problem can be regarded as a penalization of the original one. It becomes clear that this framework allows a unified perspective on several free boundary problems treated in the literature separatedly. Furthermore, it is well adapted for the numerical analysis, as it has been remarked solving particular cases (cf. [17]).

A further application is the optimal design of composite membranes, in the sense that an optimal distribution of two materials is calculated so that the least eingenfrequency (the fundamental frequency) is maximized. This was presented in [16].

\section{Statement of the problem}

Let $D \subset \mathbb{R}^{2}$ be a starshaped, bounded domain, whose boundary $\Sigma$ is a Lipschitz curve. Let $\Omega_{0} \subset D$ be a starshaped, conneted subdomain, with Lebesgue-measure $A_{0}$, whose boundary $\Gamma_{0}$ is Lipschitz-continuous. Furthermore let be given a bounded, strictly positive function $f$ defined on $D$, and let us denote as $C$ the convex set of positive, bounded functions $\mu$ such that

$$
\begin{gathered}
0 \leq \mu \leq 1 \text { almost everywhere in } D \\
\int_{\Omega_{0}} \mu d \omega=A_{0} .
\end{gathered}
$$

Besides, for a given constant $A<\operatorname{meas}(D)$ :

$$
\int_{D} \mu d \omega=A_{0}+A .
$$

We remark that conditions (2) and (3) are equivalent to

$$
\begin{aligned}
\mu & =1 \text { a.e. on } \Omega_{0} \\
\int_{D_{0}} \mu d \omega & =A \text { for } D_{0}=D-\Omega_{0} .
\end{aligned}
$$


Following the definitions introduced by Kinderlehrer-Stampacchia [19], we define for a fixed, nonnegative constant $c_{0}$ the convex set:

$$
K_{c_{0}}=\left\{\nu \in H_{0}^{1}(D) \mid \mu \geq c_{0} \text { on } \Omega_{0}\right\}
$$

where $H_{0}^{1}(D)$ denotes the usual Sobolev space and the inequality is understood in the sense of $H_{0}^{1}$.

For a fixed $\mu \in C$, we define on the Sobolev space the functional

$$
v \rightarrow J_{\mu}(v)=\frac{1}{2} \int_{D}|\nabla v|^{2} d \omega-\int_{D} \mu f v d \omega
$$

THE PROBLEM $P(\mu)$ : The minimization of $v \rightarrow J_{\mu}(v)$ on $K$ is now a classical subjet, so that for each $\mu \in L^{\infty}\left(D, \mathbb{R}^{+}\right)$there exists a $u_{\mu} \in K$ such that the functional is minimized and $u_{\mu}$ is the weak solution of the following boundary value problem $P(\mu)$ :

$$
\begin{gathered}
-\Delta u_{\mu}=\mu f \text { in } D_{0}=D-\Omega_{0} \\
u_{\mu}=c_{0} \text { on } \Omega_{0} \\
u_{\mu}=0 \text { on } \Sigma .
\end{gathered}
$$

Remark 2.1 We recall that $u_{\mu} \in C_{l o c}^{1,1}(D) \cap H^{2}(D)$ is a positive, superharmonic function.

\subsection{The optimization problem related to $\mu$}

The functional $\Phi$ defined by

$$
\mu \rightarrow \Phi(\mu)=J_{\mu}\left(u_{\mu}\right)=\min _{u \in K} J_{\mu}(u)
$$

has been studied by Gonzalez de Paz in the framework of applications to shape domain optimization (cf. [13], [15]). We have studied the minimization of $\Phi$ in $C \subset L^{\infty}\left(D, \mathbb{R}^{+}\right)$ where $C$ is the convex set defined by the constraints (2.1), (2.2) and (2.3).

Theorem 2.1 The mapping $\mu \rightarrow \Phi(\mu)$ is $\sigma\left(L^{\infty}, L^{1}\right)$-continuous on $C \subset L^{\infty}\left(D, \mathbb{R}^{+}\right)$.

PROOF: First we remark that using a-priori majorations, we can prove that there exists a ball $U \in H_{0}^{1}(D)$ such that for every $\mu \in C$, the corresponding solution $u_{\mu} \in U$. Note that using integration by parts we have:

$$
\Phi(\mu)=-\frac{1}{2} \int_{D} \mu u_{\mu} f d \omega
$$

Let us take a sequence $\left(\mu_{n}\right)_{n}$ which converges to a $\tilde{\mu} \in C$ in the topology cited above. For the sequence of the corresponding solutions $\left(u_{n}\right)_{n}$ and $\tilde{u}$ we have for every test-function $\varphi$ :

$$
\left(\nabla\left(u_{n}-\tilde{u}\right), \nabla \varphi\right)=\left(f \mu_{n}-f \tilde{\mu}, \varphi\right) .
$$


This implies that $u_{n} \stackrel{\sigma}{\rightarrow} \tilde{u}$ in $H_{0}^{1}(D)$ and due to the Rellich-Kondrasov theorem, we have also strong convergence in $L^{1}(D)$. We write now:

$$
\left\langle\mu_{n}, f u_{n}\right\rangle-\langle\tilde{\mu}, f \tilde{u}\rangle=\left\langle\mu_{n}-\tilde{\mu}, f u_{n}\right\rangle+\left\langle\tilde{\mu}, f u_{n}-f \tilde{u}\right\rangle .
$$

The brackets describe the $\left(L^{\infty}, L^{1}\right)$ - duality. In the right side of the identity, the second term converges to zero due to the strong convergence in $L^{1}(D)$, for the first one we recall that on the unit ball of $L^{\infty}$ the $\sigma\left(L^{\infty}, L^{1}\right)$ convergence is equivalent to the uniform convergence on the strongly compact subsets of $L^{1}(D)$. As every $u_{n}$ is in the ball $U$, and this is $L^{1}$-compact as a consequence of the Rellich-Kondrasov theorem, this term converges also to zero.

Remark 2.2 The above theorem can be also interpreted the following way: the corresponding Green operator defined on the set $C \subset L^{\infty}\left(D, \mathbb{R}^{+}\right)$is continuous related to the $\sigma\left(L^{\infty}, L^{1}\right)$ and the norm topology in $H_{0}^{1}(D)$ respectively.

Remark 2.3 The set $C$ is $\sigma\left(L^{\infty}, L^{1}\right)$ - compact and it has been shown that the application $\Phi$ is also continuous for the same topology, so that a minimizing element $\mu^{*}$ exists. Besides, as the lower enveloppe of affine functions relative to $\mu$, it is concave and furthermore, differentiable, the first implies that among the minimizing elements there are extremal points of $C$, wich are characteristic functions of sets with measure $A+A_{0}$. So there exists $\mu^{*}=\chi_{\tilde{\Omega}}$ with $\tilde{\Omega}=\Omega_{0} \cup \Omega$. The study of the necessary conditions of optimality furnishes the description of the boundary $\Gamma$ of $\Omega$ related to $D_{0}$ as a free boundary.

We remark that, if we define for a positive $W \in L^{1}$ :

$$
\Phi_{W}(\mu)=\Phi(\mu)+\langle W, \mu\rangle
$$

where the brackets describe the $\left(L^{1}, L^{\infty}\right)$ - duality, the functional $\Phi_{W}$ remains $\sigma\left(L^{\infty}, L^{1}\right)$ continuous and concave so that the main results remain valid, i.e. there exists an element $\mu^{*} \in C$ minimizing $\Phi_{W}$ such that $\mu^{*}=\chi_{\Omega^{*}}$ for a set $\Omega^{*}$. In physical applications, the term $\langle W, \mu\rangle$ describes a potential energy, so that $W$ might be a gravitational or electromagnetic potential function, hence harmonic.

\subsection{Necessary conditions of optimality}

Similar as in Gonzalez de Paz [13], the functional $\Phi_{W}$ is the lower enveloppe of a family of affine functions, hence concave, it follows as a consequence of a theorem due to Valadier [32]:

Theorem 2.2 The functional $\Phi_{W}$ has a weak derivative in the sense of Gateaux and its weak gradient is: $\nabla \Phi_{W}(\mu)=W-f u_{\mu}$ where $u_{\mu} \in H_{0}^{1}(D)$ is the corresponding solution of the boundary problem $P(\mu)$. So that for every $\alpha=\mu-\mu^{*}$, with $\mu \in L^{\infty}\left(D, \mathbb{R}^{+}\right)$and $\mu^{*} \in K$ solution of $P\left(\mu^{*}\right)$ we have:

$$
\Phi_{W}^{\prime}\left(\mu^{*} ; a\right)=\int_{D}\left(-u^{*} f+W\right) \alpha d \omega
$$


SKETCH OF THE PROOF: Recall that due to the definition of $\Phi_{W}$, this mapping is the lower enveloppe of affine functions related to $\mu$, thus it is a concave function. It follows from a theorem due to Valadier [32] (see also an earlierversion in Pschenichnii [26]):

$$
\Phi_{W}^{\prime}\left(\mu^{*} ; \alpha\right)=\max _{u \in \Gamma\left(\mu^{*}\right)}\langle W-f u, \alpha\rangle
$$

where $\Gamma\left(\mu^{*}\right)=\left\{u \in K / \frac{1}{2}\|\nabla u\|^{2}+\left\langle W-f u, \mu^{*}\right\rangle=\Phi\left(\mu^{*}\right)\right\}$. The energy functional is strictly convex on $K$, so it follows that the set $\Gamma\left(\mu^{*}\right)$ has only one element, namely $u^{\times}$, this means:

$$
\Phi_{W}^{\prime}\left(\mu^{*} ; \alpha\right)=\left\langle W-f u^{*}, \alpha\right\rangle .
$$

Theorem 2.3 For the optimal set $\Omega^{*}$ there exists a constant $\lambda$ such that

$$
\Omega^{*}=\left\{x \in D_{0} \mid f(x) u^{*}(x)-W(x)>\lambda\right\} .
$$

Let the functions $f$ and $W$ be continuous, then the boundary $\Gamma$ of the set $\Omega^{*}$ related to $D$ has the property: $\Gamma \subset S_{\lambda}$, where

$$
S_{\lambda}=\left\{x \in D_{0} \mid f(x) u^{\times}(x)-W(x)=\lambda\right\} .
$$

Furthermore, if $f$ is a constat and $W$ a harmonic function, then we have equality in the last relation.

PROOF: Classically, the first-order necessary condition of optimality states for every direction $\alpha=\mu-\mu^{*}$ with $\mu \in C$ :

$$
\left\langle W-f u^{*}, a\right\rangle \geq 0
$$

and for the case of characteristic functions:

$$
\int_{\Omega^{*}}\left(W-f u^{*}\right) d \omega \leq \int_{\Omega}\left(W-f u^{\times}\right) d \omega
$$

When minimizing the linear functional $\mu \rightarrow \int_{D}\left(W-f u^{*}\right) \mu d \omega$ on $C$, it follows that there exists a Lagrange multiplier related to the integral constraint (3) such that

$$
\begin{gathered}
f(x) u^{\times}(x)-W(x)>\lambda \text { implies } \mu^{*}(x)=1 \\
f(x) u^{*}(x)-W(x)=\lambda \text { implies } \mu^{*}(x) \in[0,1] \\
f(x) u^{\times}(x)-W(x)<\lambda \text { implies } \mu^{*}(x)=0 .
\end{gathered}
$$

If and $W$ are continuous, the inclusion $\Gamma \subset S_{\lambda}$ is a classical fact. If $f$ is a constant and $W$ a harmonic function, the gradient is a superharmonic function, so it follows the other inclusion (see [13]).

Furthermore, as $u^{*} \in H^{2}\left(D_{0}\right) \cap C_{l o c}^{1,1}$, the partial differential equation is verified in the a.e. sense, so it follows that the level set $S_{\lambda}=\left\{x \in D_{0} \mid f(x) u^{*}(x)-W(x)=\lambda\right\}$ has zero Lebesgue measure.

Remark 2.4 In the case $W=$ const., it can be interpreted as a Lagrange multiplier related to the measure constraint (3). In this case constraint (3) would be redundant if it were given explicitly. 


\section{An application in steady vortex flow}

Let $D \subset \mathbb{R}^{2}$ be a bounded domain, shaped as a stripe with height $A$ and length $2 L$, in fact the domain we have in mind is the cartesian product $[-L, L] \times[0, A]$. We note its boundary as $\Sigma$. Let $\Omega_{0} \subset D$ be a domain with a smooth boundary $\Gamma_{0}$ describing a body in the domain $D$, which is occupied by an ideal fluid. We shall assume that the center of the stripe lies somewhere in $\Omega_{0}$. For $D_{0}=D-\Omega_{0}$ we define $\eta$ such that

$$
\begin{gathered}
\Delta \eta=0 \text { in } D_{0} \\
\eta=0 \text { on } \Gamma_{0} \\
\eta=y \text { on } \Sigma,
\end{gathered}
$$

the function $\eta$ is the stream-function for a normalized irrotational flow in $D_{0}$.

Recall that the vorticity field $\vec{w}=\nabla \times \vec{v}$ can be written in this case as $\vec{w}=\zeta \vec{i}_{z}$ where $\zeta$ is called the vorticity function and $\vec{v}=\left(\frac{\partial \tilde{\psi}}{\partial y},-\frac{\partial \tilde{\psi}}{\partial x}\right)$ for some stream-function $\tilde{\psi}$. Finally we have that:

$$
-\Delta \tilde{\psi}=\zeta \text { in } D
$$

This motivates the following definition: for a given positive, bounded function $\zeta$ we define on the Sobolev space $H_{0}^{1}\left(D_{0}\right)$ the functional

$$
\psi \rightarrow J_{\zeta}(\psi)=\frac{1}{2} \int_{D_{0}}|\nabla \psi|^{2} d x-\int_{D_{0}} \zeta \psi d x
$$

Classically, this functional has a minimizing element in $H_{0}^{1}\left(D_{0}\right)$ which is a weak solution of the Poisson equation (4) defined above. Furthermore, we are able to define on $L^{\infty}\left(D_{0}, \mathbb{R}^{+}\right)$ the functional:

$$
\zeta \rightarrow \Phi(\zeta)=\min _{\psi \in H_{0}^{1}} J_{\zeta}(\psi)
$$

We remark that for $\psi_{\zeta} \in H_{0}^{1}(D)$ minimizing element for a fixed $\zeta$, when applying integration by parts, we obtain for the kinetic energy of the flow:

$$
E(\zeta)=\frac{1}{2} \int_{D_{0}}\left|\nabla \psi_{\zeta}\right|^{2} d x=\frac{1}{2} \int_{D_{0}} \psi_{\zeta} \zeta d x=-\Phi(\zeta)
$$

Besides, following the definition presented by Turkington [31] the vortex impulse (i.e. the total impulse required to generate the flow from rest), is given by the linear functional:

$$
\zeta \rightarrow \int_{D_{0}} \eta \zeta d x
$$

We define now for a positive scalar $W$ the functional

$$
\Phi_{W}(\zeta)=\Phi(\zeta)+W \int_{D_{0}} \zeta \eta d x
$$

In fact, $W$ describes the speed of the fluid on $\Sigma$ in the case there is not a vortex. For the case that the stripe is very long related to the body $\Omega_{0}$, this means that far from the obstacle the modulus of velocity of the fluid tends to the constant $W$. Furthermore we define as $C$ the convex subset in $L^{\infty}$ of functions $\zeta$ with the following constraints: 
1. For a fixed $\beta$

$$
0 \leq \zeta(x) \leq \beta \text { a.e. in } D_{0}
$$

the constant $\beta$ is related to the vortex intensity.

2. For a fixed, positive constant $\Gamma$, which describes the total circulation of the vortex:

$$
\int_{D_{0}} \zeta(x) d x=\Gamma
$$

As a technical condition which will be explained in the next section, we assume:

$$
\frac{\Gamma}{\beta} \leq \operatorname{meas}\left(D_{0}\right)
$$

Other authors have studied the maximization of the functional $\zeta \rightarrow E(\zeta)$ by using rearrangemensts of functions. Here our aim will be to study the minimization of $\Phi_{W}$ on $C$ using Convex Analysis techniques.

Theorem 3.1 The functional $\Phi_{W}$ is concave and $\sigma\left(L^{\infty}, L^{1}\right)$-continuous, so that there exists an element $\zeta^{*} \in C$ such that

$$
\Phi_{W}\left(\zeta^{*}\right)=\min _{\zeta \in C} \Phi_{W}(\zeta)
$$

Remark 3.1 As the functional $\Phi_{W}$ is concave, among its minimizing points there are extremal points of $C$. They are of the form $\zeta=\beta \chi_{\Omega}$ where $\chi_{\Omega}$ is a characteristic function of a set $\Omega$. Consequently, the constraints (26) and (27) become

$$
\operatorname{meas}(\Omega)=\frac{\Gamma}{\beta} \leq \operatorname{meas}\left(D_{0}\right) \text {. }
$$

Consequently, in order that the model be physically meaningful, the constants $A$ and $L$ shall be assumed large enough in order that for a fixed $\Gamma$ a broad range of values for the parameter $\beta$ may be feasible.

As a consequence of Theorem 2.3:

Theorem 3.2 Let $\psi^{*}$ be the solution for $\zeta^{*}$. For the optimal set $\Omega^{*}$ there exists a constant $k$ such that

$$
\begin{aligned}
& \Omega^{*}=\left\{x \in D_{0} \mid \psi^{*}(x)-W \eta(x)>k\right\} \\
& \partial \Omega^{*}=\left\{x \in D_{0} \mid \psi^{*}(x)-W \eta(x)=k\right\}
\end{aligned}
$$

Remark 3.2 As we can see, the function $\psi^{*} \in H_{0}^{1}\left(D_{0}\right)$ is a solution of the free boundary value problem

$$
\begin{gathered}
-\Delta \psi^{*}=\beta \text { in } \Omega^{\times} \\
\Delta \psi^{*}=0 \text { in } D_{0}-\Omega^{*} \\
\psi^{*}=k+W \eta \text { on } \partial \Omega^{*}
\end{gathered}
$$


Remark 3.3 Because of the known regularity of $\psi^{*}$, the gradient is continuous across the free boundary $\partial \Omega^{*}$. It is known that free boundaries of this type are locally Lipschitz (cf. Kinderleherer-Stampacchia [19]) and even locally $C^{1, \alpha}$. So we may write as an additional property:

$$
\left(\nabla \psi^{*}\right)^{+}=\left(\nabla \psi^{*}\right)^{-} \text {on } \partial \Omega^{\times} .
$$

Here the plus sign denotes the limit at the boundary takes in the inward direction to $\Omega^{*}$ and the minus sign denotes the limit in the outward direction.

Remark 3.4 We are able to define now the adjusted stream function:

$$
\tilde{\psi}=\psi^{\times}-W \eta-k
$$

Note that in this case the stream function $\tilde{\psi}>0$ in the domain $\Omega^{*}$ where we have the vortex and $\tilde{\psi}=0$ on the free boundary $\partial \Omega^{*}$. Besides,

$$
\begin{gathered}
-\Delta \tilde{\psi}=\zeta \text { in } D_{0} \\
\tilde{\psi}=-k \text { on } \Gamma_{0} \\
\tilde{\psi}=-W \eta-k \text { on } \Sigma .
\end{gathered}
$$

The term $u=\psi^{*}-k$ can be interpreted as the perturbation of the stream function due to the vortex.

\section{Shape optimization problems in classical potential theory}

We consider the classical capacity problem: given a doubly connected domain $\Omega \subset \mathbb{R}^{2}$, we denote by $\Gamma_{0}$ and by $\Gamma$ the interior and exterior boundary of $\Omega$ respectively, and by $\Omega_{0}$ the domain bounded by $\Gamma_{0}$. Classically, a potential function $u_{\Omega}$ solves the Dirichlet boundary value problem for the Laplace equation in $\Omega$ :

$$
\begin{aligned}
& \Delta u_{\Omega}=0 \text { in } \Omega \\
& u_{\Omega}=1 \text { on } \Gamma_{0} .
\end{aligned}
$$

The capacity of the domain $\Omega_{0}$ related to $\Omega$, noted $\operatorname{Cap}_{\Omega}\left(\Omega_{0}\right)$ is given by:

$$
\operatorname{Cap}_{\Omega}\left(\Omega_{0}\right)=\int_{\Omega}\left|\nabla_{\Omega}\right|^{2} d \omega
$$

Let be given the following isoperimetric condition: $\operatorname{meas}(\Omega)=A$ with $A$ a positive constant. It is a well known result that the capacity is minimized when the shape of $\Omega$ is a circular ring. We shall deal here with the question how to optimize the shape of $\Omega$ in the sense that the capacity is minimized, when either the interior boundary $\Gamma_{0}$ or the exterior boundary $\Gamma$ is a given, fixed Lipschitz curve. 


\subsection{The exterior boundary case}

We shall deal firstly with a relaxed problem: assume that the domain $D$ is a ball of radius $R$ (i.e. $D=B_{R}$ ), with its center located somewhere in the interior of $\Omega_{0}$. The radius $R$ is chosen "large" enough. For a given bounded $f>0$ and fixed $\mu \in C$ we minimize the functional $u \rightarrow J_{\mu}(u)$ on $K_{1}$. For a given $f$ we consider the minimization of the energy functional $\mu \rightarrow \Phi(\mu)$ on $C$. As proved before, there exists a minimizing element $\mu_{R} \in C$ and a corresponding solution: $u_{R} \in H_{0}^{1}\left(B_{R}\right)$ of $P\left(\mu_{R}\right)$. From the necessary conditions of optimality, in the case that $f$ is constant and $W$ is harmonic, applying Theorem 2.3 it follows that $\mu_{R}$ is the characteristic function of a set

$$
\Omega_{R}=\left\{x \in B_{R} \mid f(x) u_{R}(x)-W(x)>p_{R}\right\}
$$

and

$$
\Gamma=\partial \Omega_{R} \cap D=\left\{x \in B_{R} \mid f(x) u_{R}(x)-W(x)=p_{R}\right\} .
$$

The constant $p_{R}$ may by interpreted as a Lagrange multiplier related to the area constraint (3). Under the hypothesis that there exists a ball $B_{0}$ large enough, so that for every $R$ of an increasing sequence converging to infinity: $\Omega_{R} \subset B_{0}$, it has been shown in Gonzalez de Paz [15]:

Theorem 4.1 In the case of an increasing sequence of radius $R$, such that its limit is in infinity, there exists a sequence of minimizing elements $\left(\mu_{R}\right)_{R}$ in $L^{\infty}\left(\mathbb{R}^{2}, \mathbb{R}^{+}\right)$and a corresponding sequence of solutions $\left(u_{R}\right)_{R}$ such that for the canonical extensions $\tilde{u}_{R} \in H^{1}\left(\mathbb{R}^{2}\right)$ to the whole space we have:

$$
\begin{gathered}
\tilde{u}_{R} \rightarrow u^{*} \text { strongly in } H^{1}\left(\mathbb{R}^{2}\right) \\
\mu_{R} \rightarrow \mu^{*}=\chi_{\Omega^{*}} \text { strongly in } L^{2}\left(\mathbb{R}^{2}\right)
\end{gathered}
$$

where $\Omega^{*}=\left\{x \in \mathbb{R}^{2} \mid u^{\times}(x)>0\right.$ in the $H^{1}$-sense $\}$. Besides,

$$
\Phi\left(\mu_{R}\right) \rightarrow \Phi\left(\mu^{*}\right)=\frac{1}{2} \int_{\Omega^{*}}\left|\nabla u^{*}\right|^{2} d \omega-\int_{\Omega^{*}} f u^{*} d \omega+\int_{\Omega^{*}} W d \omega
$$

such that for every $R$ :

$$
\Phi\left(\mu^{*}\right) \leq \Phi\left(\mu_{R}\right)
$$

and for $\Omega^{*}$ regular enough:

$$
-\Delta u^{*}=f \text { in } \Omega^{\times}
$$

Remark 4.1 In fact, the element $u^{*} \in H^{1}\left(\mathbb{R}^{2}\right)$ solves a related free boundary value problem studied by H. Alt and L. Cafarelli (cf. [1]) and we remark that, under certain hypothesis concerning the regularity of the boundary $\Gamma_{0}$ (namely, locally Lipschitz), those authors have proved that the free boundary $\Gamma$ is locally of the type $C^{1, \alpha}$ and even locally analytic. 
Remark 4.2 Again, let us consider the case $W=0$ and $f=$ constant. It has been proved that in this case, the set $\Omega^{*}$ cited in the Theorem above has the property that, for every connected domain $\Omega$ such that $\Omega_{0} \subset \Omega$, meas $(\Omega)=\operatorname{meas}\left(\Omega^{*}\right)$ :

$$
\operatorname{cap}_{\Omega^{*}}\left(\Omega_{0}\right) \leq \operatorname{cap}_{\Omega}\left(\Omega_{0)}\right.
$$

Remark 4.3 The same method has been applied in a particular problem of elasticity theory: we consider the elastic torsion of a hollow elastic shaft, in this case $\Omega_{0}$ denotes the hole cross section of the shaft. Assuming that the boundary $\Gamma_{0}$ and the measure of $\Omega$ are given, we look for the shape of $\Omega$ such that the torsional stiffness

$$
K_{\Omega}=2\left(\int_{\Omega} u d \omega+c A_{0}\right)
$$

is maximized

It is well known that the stress function $u$ solves the following boundary value problem:

$$
\begin{gathered}
-\Delta u=2 \text { in } \Omega \\
u=0 \text { on } \Gamma \\
u=c \text { on } \Gamma_{0} \\
-\int_{\Gamma_{0}} \frac{\partial u}{\partial n} d s=2 A_{0}
\end{gathered}
$$

where $A_{0}$ is the area of the region bounded by $\Gamma_{0}$ and $c$ is an unknown quantity whose value can be determined using (46). Using a relaxed problem defined on a ball $B_{R}$ as before, the existence of an optimal domain $\Omega_{R}$ has been proved in [14]. In [22], Lederman and in [30] R. Tahraoui prove that the domain $\Omega_{R}$ is connected and, for a radius $R$ large enough, it solves the original problem. If $\Gamma_{0}$ is $C^{2}$, C. Lederman [22] has recently proved that the exterior boundary $\Gamma$ of the optimal set $\Omega_{R}$ is locally analytic.

\subsection{The interior boundary case}

Let the boundary $\Gamma$ and the area $\left|\Omega_{0}\right|=A_{0}$ be given, we look for the shape of the interior boundary $\Gamma_{0}$ in order to minimize the capacity $\operatorname{Cap}_{\Omega}\left(\Omega_{0}\right)$. An important physical interpretation is the following: If $D$ is filled with a homogenous, isotropic material we look how to locate and how to shape a heat source within $D$ such that the heat leakage from the body $D$ is minimized. The boundary condition on the inner domain $\Omega_{0}$ will be treated via a penalization term. Let $\mu$ be a bounded non-negative function (i.e. $0 \leq \mu \leq 1$ ) such that: $\int_{D} \mu d \omega=A$. For a fixed $\mu$ as defined and a fixed, positive constant $\beta$ we define the functional on $H_{0}^{1}(D)$ :

$$
u \rightarrow J_{\beta}(u)=\|\nabla u\|^{2}+\beta \int_{D} \mu|u-1|^{2} d \omega
$$

Applying standard properties of Variational Analysis, the following result is valid: 
Theorem 4.2 There exists only one element $u_{\beta}(\mu) \in H_{0}^{1}(D) \cap C^{1, \alpha}(D)$ such that the functional $J_{\beta}$ is minimized. Furthermore, $u_{\beta}$ solves the partial diferential equation:

$$
-\Delta u+\beta \mu(u-1)=0 \text { in } D .
$$

Again, we define the mapping $\mu \rightarrow \Phi(\mu)=J_{\beta}\left(u_{\beta}(\mu)\right)$ on the convex set $C=\left\{\mu \in L^{\infty} \mid 0 \leq \mu \leq 1, \int \mu d \omega=A\right\}$, and when considering its minimization on $C$, we obtain the usual result:

Theorem 4.3 There exists a set $\Omega_{\beta} \subset D$ such that the function $\mu_{\beta}=\chi_{\Omega}$ minimizes $\Phi$ on $C$. Furthermore, there exists a constant $p_{\beta}$ so that

$$
\begin{gathered}
\Omega_{\beta}=\left\{x \in D \mid u_{\beta}(x)>p_{\beta}\right\} \\
\partial \Omega_{\beta}=\left\{x \in D \mid u_{\beta}(x)=p_{\beta}\right\} .
\end{gathered}
$$

Remark 4.4 For the corresponding element $u_{\beta} \in H_{0}^{1}(D)$ this means:

$$
\begin{gathered}
-\Delta u_{\beta}+\beta\left(u_{\beta}-1\right)=0 \text { in } \Omega_{\beta} \\
\Delta u_{\beta}=0 \text { in } D-\Omega_{\beta} .
\end{gathered}
$$

It is known that free boundaries in this kind of problems are locally analytic.

It has been shown in Gonzalez de Paz [13]:

Theorem 4.4 In the case there is an increasing sequence $\{\beta\}$ tending to infinity, there exists a subsequence so that for the corresponding sequences $\left\{u_{\beta}\right\},\left\{\mu_{\beta}\right\}$ :

$$
\begin{aligned}
& u_{\beta} \rightarrow u^{*} \text { in } H_{0}^{1}(D) \\
& \mu_{\beta} \rightarrow \mu^{*} \text { in } L^{2}(D) .
\end{aligned}
$$

Furthermore, $\mu^{*} \in C$ is the characteristic function of a set $\Omega^{\times}$such that $u^{*}=1$ on $\Omega^{*}$ and for $\partial \Omega^{*}$ regular enough $u^{*}$ is harmonic in $\Omega$, so that in this case for every $\Omega_{0}$ such that $\left|\Omega_{0}\right|=A$ :

$$
\operatorname{Cap}_{\Omega}\left(\Omega^{*}\right) \leq \operatorname{Cap}_{\Omega}\left(\Omega_{0}\right)
$$

Remark 4.5 As $u^{*} \in H_{0}^{1}$ solves a variational problem similar to those studied by AltCafarelli[1] the free boundary $\partial \Omega^{*}$ has a $C_{l o c}^{1, a}$-regularity.

Remark 4.6 Let us remark that in the distributions-sense:

$$
-\Delta u^{*}=\nu^{*} \text { in } D
$$

for a finite energy measure $\nu^{\times}$such that its support in the distribution sense lies in $\partial \Omega^{*}$. 


\section{$5 \quad$ Free boundary value conditions}

\subsection{Calculation of the shape-derivative}

Let us consider again the exterior boundary case for the shape optimization problem. Let $\phi_{t}: D \rightarrow D$ be a diffeomorphism which is continuously dependent of a positive parameter $t$, so that $\phi_{0}$ is the identity mapping (i.e. for any domain $\Omega \subset D: \phi_{0}(\Omega)=\Omega$ ). Furthermore, for a given domain $\Omega \subset D$ we denote $\phi_{t}(\Omega)=\Omega_{t}$, so that the mapping $t \rightarrow \phi_{t}$ describes continuous deformations of $\Omega$. Using a certain topology, J.P. Zolesio in [34] calculates the derivative $D_{t} \phi_{t}=\theta$ where $\theta$ is the vector field describing the "deformation speed". For the domain functional $\Omega_{t} \rightarrow J\left(\Omega_{t}\right)$, the shape-derivative at $\Omega$ in the direction of the vector field $\theta$ is defined in the sense of Eulerian semiderivatives as follows (for futher details see for example M. Delfour [7]):

$$
d J(\Omega ; \theta)=\lim \sup _{t \rightarrow 0^{+}} \frac{J\left(\Omega_{t}\right)-J(\Omega)}{t} .
$$

Applying the techniques developed by Sokolowski-Zolesio [28] it has been shown that in the case that the function $u_{0}$ solution for $P\left(\mu_{0}\right)$ is constant on $\Gamma$, then we have for small $t$ and $\alpha_{t}=\chi_{\Omega_{t}}-\chi_{\Omega}$ :

$$
\Phi\left(\mu_{t}\right)=\Phi\left(\mu_{0}\right)+\left\langle W-f u_{0} ; \alpha_{t}\right\rangle+\frac{t}{2} \int_{\Gamma}\left(\left|\frac{\partial u_{0}^{+}}{\partial n}\right|^{2}-\left|\frac{\partial u_{0}^{-}}{\partial n}\right|^{2}\right) \theta_{n} d s
$$

where the restrictions of $u_{0}$ to $\Omega$ and $D-\Omega$ are denoted by $u_{0}^{+}$and $u_{0}^{-}$respectively, $\mu_{t}=\chi_{\Omega_{t}}, \mu_{0}=\chi_{\Omega}$ and the term $\theta_{n}$ describes the normal component to $\Gamma$ of the vector field $\theta$.

In fact, in the particular case of the relaxed problem the last integral term of the right side vanishes because of the "transmission" condition [35] and we obtain as functional gradient the same term as Theorem (2.2). We remark that in the case that the function $u_{0}$ is not constant on $\Gamma$ we shall have non-zero tangential derivatives of $u_{0}$ on $\Gamma$.

Remark 5.1 Let $D=\mathbb{R}^{2}$. Let us consider the limit case for the outer boundary problem. We recall that, due to the properties of the logarithmic potential in $\mathbb{R}^{2}$, as $u^{*}$ is harmonic and bounded in the complement of $\Omega^{*} \cup \Omega_{0}$ which is an unbounded domain, then $\mu^{*}$ is zero on it. The gradient $\nabla u^{*}$ is not a continuous function anymore, so that in the case that the corresponding boundary $\Gamma$ is regular enough, and taking notice that $u^{*}=0$ on it, the shape derivative becomes:

$$
d \Phi(\Omega, \theta)=\int_{\Gamma}\left(W-\frac{1}{2}\left|\frac{\partial u^{-}}{\partial n}\right|^{2}\right) \theta_{n} d s .
$$

As $d \Phi\left(\Omega^{*}, \theta\right)=0$ for every vector field $\theta$ such that the measure is preserved, (i.e. $\left.\int_{\Gamma} \theta_{n} d s=0\right)$ it follows that there exists a constant $\lambda$ such that:

$$
W-\frac{1}{2}\left|\frac{\partial u^{-}}{\partial n}\right|^{2}=\lambda \text { on } \Gamma .
$$


This boundary condition is known as a Bernoulli-condition. The special case where $W=Q^{2}$ and there is no constraint of type (3), has been treated in the famous paper by Alt-Cafarelli [1]. In this case the Bernoulli condition becomes:

$$
\frac{1}{2}\left|\frac{\partial u^{-}}{\partial n}\right|^{2}=Q^{2} \text { on } \Gamma
$$

\subsection{The non-linear Bernoulli condition}

Some attention has been drawn lately on problems with non linear conditions of the type

$$
-\left|\frac{\partial u}{\partial n}\right|^{2}+W+\sigma H=\lambda
$$

on the free boundary $\Gamma$, where $\sigma>0$ is a constant and $H(x)$ describes the curvature of the boundary (see for instance, J.P. Zolesio [36], and M. Visintin [33]). We shall review the main results known for this case. The main difference lies in the fact that we consider the minimization on $C$ of functionals of the type:

$$
\Phi_{\sigma}(\mu)=\Phi_{W}(\mu)+\sigma \Lambda(\mu)
$$

where the functional $\Lambda$ is defined as follows:

$$
\mu \rightarrow \Lambda(\mu)=\|\nabla \mu\|_{M_{0(D)}}=\sup _{g \in B_{0}} \int_{D} \mu \operatorname{div} g d \omega
$$

where $B_{0}=\left\{g \in C_{0}^{1}\left(D, R^{2}\right)|0 \leq| g(x) \mid \leq 1\right\}$ and $M_{0}$ is the set of bounded measures on $D$ (cf. [11]). It is known that for the case that $\mu=\chi_{\Omega}$ for a set $\Omega$ with a regular enough boundary, $\Lambda\left(\chi_{\Omega}\right)$ describes the perimeter of the set $\Omega$ in $D$. In the fluid mechanics context, $\sigma \Lambda(\mu)$ represents an energy term due to surface tension. Working with this kind of functionals, an additional constraint for the perimeter $P(\Omega)$ is given. This allows to use well-known compacity results for the characteristic functions in order to prove the existence of optimal domains $\Omega$ constraining within sets of characteristic functions (see Ambrosio-Buttazzo [2], Zolesio [36]). We will show that these cases may be considered as penalizations of the energy functionals already studied.

The functional $\mu \rightarrow \Lambda(\mu)$ is convex and $\sigma\left(L^{\infty}, L^{1}\right)$-lower-semi-continuous. For a positive constant $\sigma$, the functional $\Phi_{\sigma}$ is quasiconcave and $\sigma\left(L^{\infty}, L^{1}\right)$-l.s.c. If we consider the minimization of $\Phi_{\sigma}$ on $C$ as before, for every $\sigma$ there exists a $\mu_{\sigma} \in C \cap B V(D)$ such that the functional is minimized, here $B V(D)$ denotes the set of measures with bounded variation (see Giusti [11]). We can prove the following:

Theorem 5.1 For each positive $\sigma$, there exists a set $\Omega_{\sigma}$ with finite perimeter such that $\mu_{\sigma}=\chi_{\Omega}$

PROOF: As $\mu_{\sigma} \in C$ there exist extremal points $\mu_{1}, \mu_{2}$ of $C$ such that : $\mu_{\sigma}=\alpha \mu_{1}+(1-\alpha) \mu_{2}$ for some $\alpha \in[0,1]$. It follows that

$$
\Phi_{\sigma}\left(\mu_{\sigma}\right) \leq \Phi_{\sigma}\left(\mu_{1}\right), \Phi_{\sigma}\left(\mu_{\sigma}\right) \leq \Phi_{\sigma}\left(\mu_{2}\right) .
$$


Let us assume that $\Phi\left(\mu_{1}\right) \leq \Phi\left(\mu_{2}\right)$, then, as $\Phi_{\sigma}$ is quasiconcave it follows that for every $\alpha \in[0,1]$ :

$$
\Phi_{\sigma}\left(\mu_{1}\right) \leq \Phi_{\sigma}\left(\alpha \mu_{1}+(1-\alpha) \mu_{2}\right)
$$

this implies:

$$
\Phi_{\sigma}\left(\mu_{1}\right) \leq \Phi_{\sigma}\left(\mu_{\sigma}\right)
$$

so we conclude that: $\Phi_{\sigma}\left(\mu_{1}\right)=\Phi_{\sigma}\left(\mu_{\sigma}\right)$ and this allows to choose $\mu_{\sigma}$ as an extremal point of $C$, wich is a characteristic function.

Remark 5.2 Noting that when we exchange the order of minimization in our problem, we obtain that for every $u \in K$ the optimal $\mu_{\sigma}$ solves the following:

$$
\min _{\mu \in C}\langle W-f u, \mu\rangle+\sigma \Lambda(\mu)
$$

more specially, for $u=u_{\sigma}$ corresponding solution for $P\left(\mu_{\sigma}\right)$ :

$$
\left\langle W-f u_{\sigma}, \mu_{\sigma}\right\rangle+\sigma \Lambda\left(\mu_{\sigma}\right) \leq\left\langle W-f u_{\sigma}, \mu\right\rangle+\sigma \Lambda(\mu)
$$

for every $\mu \in C$.

In the case $\mu=\chi_{\Omega}$, with $\Omega$ regular enough, Zolesio [36] has calculated the shapederivative of the functional $\Lambda$ in the sense that:

$$
d \Lambda(\Omega ; \theta)=\lim \inf _{t \rightarrow 0^{+}} \frac{1}{t}\left\{\Lambda\left(\mu_{t}\right)-\Lambda\left(\mu_{0}\right)\right\}=\langle\mathcal{H}, \theta\rangle_{\mathcal{D}^{\prime} \times \mathcal{D}}
$$

where $\mu_{t}=\chi_{\Omega}$, and $\mathcal{H} \in \mathcal{E}^{\prime}$ is a distribution such that supp $\mathcal{H} \subset \Gamma$, so that if $\Gamma$ is smooth enough:

$$
d \Lambda(\Omega ; \theta)=\int_{\Gamma} H \theta_{n} d s
$$

where $H$ is the curvature function of the boundary $\Gamma$. So, in the case that the domain $\Omega_{\sigma}$ has a boundary regular enough, it is possible to calculate the shape derivative of the functional $\mu \rightarrow \Lambda(\mu)$ and we get:

$$
d \Phi\left(\Omega_{\sigma}, \theta\right)=\int_{\Gamma}\left(W-f u_{\sigma}+\sigma H\right) \theta_{n} d s .
$$

As a consequence of the optimality conditions, this implies that there exists a Lagrange multiplier $\lambda$ related to constraint (3) such that:

$$
W-f u_{\sigma}+\sigma H=\lambda \text { on } \Gamma .
$$

We remark that in fluid mechanics, if we let $f=\epsilon \rightarrow 0^{+}$we become the well-known free boundary condition for hydrostatic problems in the case of irrotational ideal fluids:

$$
W+\sigma H=\lambda \text { on } \Gamma \text {. }
$$

Remark 5.3 It has been proved by Gonzales, Massari and Tamarini [12] that for the case of a set minimizing perimeter under area constraint the free boundary $\Gamma$ is locally analytic. 
Remark 5.4 Again, assuming that $D=B_{R}$ and letting $R \rightarrow \infty$, for the limit function we obtain $u_{\sigma}=0$ in the unbounded domain and we have for the boundary $\Gamma$ of a limit set $\Omega^{*}$ regular enough:

$$
-\frac{1}{2}\left|\frac{\partial u_{\sigma}^{-}}{\partial n}\right|^{2}+\sigma H+W=\lambda \text { on } \Gamma .
$$

Remark 5.5 The term $\sigma \lambda$ might be interpreted as a "penalization" or "regularization" term. Let us assume that $\mu^{*} \in C \cap B V(D)$ is an optimizing element for $\Phi$, (without loss of generality, let $W=0$ ). This implies, for $\sigma>0$ :

$$
\Phi\left(\mu_{\sigma}\right)+\sigma \Lambda\left(\mu_{\sigma}\right) \leq \Phi\left(\mu^{*}\right)+\sigma \Lambda\left(\mu^{*}\right)
$$

so it follows:

$$
0 \leq \Phi\left(\mu_{\sigma}\right)-\Phi\left(\mu^{*}\right) \leq \sigma\left(\Lambda\left(\mu^{*}\right)-\Lambda\left(\mu_{\sigma}\right)\right)
$$

As $\sigma>0$ :

$$
\Lambda\left(\mu_{\sigma}\right) \leq \Lambda\left(\mu^{*}\right)
$$

i.e. the terms $\Lambda\left(\mu_{\sigma}\right)$ remain bounded. Let us take for the parameter $\sigma$ a decreasing sequence such that $\sigma \searrow 0$, then it is possible to choose a subsequence $\left(\mu_{\sigma}\right)_{\sigma}$ such that $\mu_{\sigma} \rightarrow \mu_{0} \in C \cap B V(D)$ for the strong $L^{1}$-topology. The limit element $\mu_{0}$ is a characteristic function (cf. [11]). It follows from the Theorem 2.1: $\Phi\left(\mu_{\sigma}\right) \rightarrow \Phi\left(\mu_{0}\right)$ and furthermore, due to the lower semicontinuity of $\Lambda$ :

$$
\Lambda\left(\mu_{0}\right) \leq \lim \inf \Lambda\left(\mu_{\sigma}\right) \leq \Lambda\left(\mu^{*}\right)
$$

We remark that the sequence $\left(\Phi_{\sigma}\left(\mu_{\sigma}\right)\right)_{\sigma}$ is also decreasing, thus for $\sigma<\sigma^{\prime}$ we have:

$$
\Phi\left(\mu_{\sigma}\right)+\sigma \Lambda\left(\mu_{\sigma}\right) \leq \Phi\left(\mu_{\sigma^{\prime}}\right)+\sigma \Lambda\left(\mu_{\sigma^{\prime}}\right) \leq \Phi\left(\mu_{\sigma^{\prime}}\right)+\sigma^{\prime} \Lambda\left(\mu_{\sigma^{\prime}}\right) .
$$

This implies that: $\Phi_{\sigma}\left(\mu_{\sigma}\right) \searrow \Phi\left(\mu_{0}\right)$, and as for every $\sigma>0$ :

$$
\Phi\left(\mu_{0}\right) \leq \Phi_{\sigma}\left(\mu_{\sigma}\right) \leq \Phi_{\sigma}\left(\mu^{*}\right)
$$

it follows that: $\Phi\left(\mu_{0}\right) \leq \Phi\left(\mu^{*}\right)$, and as $\mu_{0} \in C$, it follows the equality. This implies that $\mu_{0}$ is an optimizing element of the original functional $\Phi$.

\section{Conclusions}

It has been shown that several well known free boundary value problems can be studied by means of a relaxation approach in the framework of Convex Analysis. All of them present common features, so that a unified theory seems well-adapted. Its main theoretical advantange lies in bridging the gap between relaxation methods and the work developed by the shape sensitivity school and it is also well-fitted for other applications in plasma physics, fluid mechanics and electrochemistry.

For the numerical solution of this kind of problems, an algorithm of the Frank-Wolfe type was presented by Gonzalez de Paz-Tiihonen [17]. Its main advantage would be that the algorithm can change the topological structure of the solution during the optimization process. Besides, there is no need to change the discretization grid, which remains fixed during the iteration process. As this kind of problems are non-convex, the algorithm allows to try different initial designs in order to compare results. 


\section{References}

[1] Alt, H.; Cafarelli, L. (1981) "Existence and regularity for a minimum problem with free boundary", J. Reine u. Angew. Mathematik 325: 104-144.

[2] Ambrosio, L.; Butazzo, G. (1993) "An optimal design problem with perimeter penalization", Calc. of Var. 1: 55-69.

[3] Butazzo, G.; Dal Maso, G. (1990) "Shape optimization for Dirichlet problems: relaxed solutions and optimality conditions", Bull. AMS 23: 531-535.

[4] Cafarelli, L. (1989) "Free boundary problems, a survey in topics in calculus of variations", M. Giaquinta (Ed.), Lect. Notes Math., Springer, New York.

[5] Cea, J.; Malanowski, K. (1970) "An example of a max-min problem in partial differential equations", SIAM J. on Control and Optimization 8: 305-316.

[6] Chenais, D. (1975) On the existence of a solution in a domain identification problem", J. Math. Anal. Appl. 52: 189-219.

[7] Delfour, M. (1992) "Shape derivatives and differentiability of min-max", in: Proceedings NATO-Université de Montréal Seminar on Shape Optimization and Free Boundaries, M. Delfour \& G. Sabidussi (Eds.), Kluwer, Dordrecht.

[8] Ekeland, I.; Teman, R. (1974) Analyse Convexe et Problèmes Variationnelles. Dunod, Paris.

[9] Friedman, A.; Turkington, B. (1981) "Vortex rings: existence and asymptotic estimates", Trans. AMS 268: 1-37.

[10] Fujii, N. (1986) "Necessary conditions for a domain optimization problem in elliptic boundary value problems", SIAM J. Control and Optimization 24: 346-360

[11] Giusti, E. (1984) Minimal Surfaces and Functions of Bounded Variation. Birkhauser, Basel.

[12] Gonzales, N.; Massari, U.; Tamarini, I. (1983) "On the regularity of boundaries of sets minimizing perimeter with a volume constraint", Indiana Univ. Math. J. 32: $25-37$.

[13] González de Paz, R.B. (1982) "Sur un problème d'optimisation de domaine", Numer. Funct. Anal. and Optimiz. 5: 173-197.

[14] González de Paz, R.B. (1989) "On the optimal design of elastic shafts", Math. Modelling and Numer. Anal. (M2AN) 23: 615-625.

[15] González de Paz, R.B. (1994) "A relaxation approach applied for domain optimization", SIAM Journal on Control and Optimization 32: 154-169. 
[16] González de Paz, R.B. "On the maximization of membrane frequencies: an eigenvalue control problem", to appear in Numer. Func. Anal. and Optimiz.

[17] González de Paz, R.B.; Tiihonen, T. (1994) "A relaxation-based numerical method applied to domain optimization", in: Proceedings Conference FEM 50, Finite Element Methods, M. Krizek, P. Neittaanmaki \& R. Stenberg (Eds.), Marcel Dekker.

[18] Jensen, R. (1980) "Boundary regularity for variational inequalities", Indiana Univ. Math. J. 29: 495-511.

[19] Kinderlehrer, H.; Stampacchia, G. (1980) An introduction to Variational Inequalities and their Applications. Academic Press, New York.

[20] Kohn, R.V. (1992) "Numerical structural optimization via a relaxed formulation", in: Proceedings NATO- Université de Montréal Seminar on Shape Optimization and Free Boundaries M. Delfour \& G. Sabidussi (Eds.), Kluwer, Dordrecht.

[21] Kohn, R.V.; Strang, G. (1986) "Optimal design and relaxation of variational problems I-II", Comm. Pure and Appl. Math. 39: 113-377.

[22] Lederman, C. (1995) "An optimization problem in elasticity", Differential and Integral Equations 8: 2025-2044.

[23] Murat, F.; Simon, J. (1974) "Quelques résultats sur le contrôle par un domaine géométrique", Rapport de Recherche No. 74-003, Lab. Analyse Numérique, Université Paris VI.

[24] Murat, F.; Tartar, J.L. (1984) Calcul de Variations et Homogénéisation. Cours Ecole d'Eté d'Analyse Numérique, CEA-EDF-INRIA, Eyrolles, Paris.

[25] Pironneau, O. (1984) Optimal Shape Desing for Elliptic Systems. Springer, Berlin.

[26] Pschenichnii, B.N. (1966) "Linear optimal control problems", J. SIAM Control 4: 577-593.

[27] Simon, J. (1980) "Differentiation with respect to the domain in boundary value problems", Numer. Funct. Anal. Optim. 2: 649-687.

[28] Sokolowski, J.; Zolesio, J.P. (1992) Introduction to Shape Optimization. Springer, Berlin.

[29] Tahraoui, R. (1992) "Contrôle optimal dans les équations elliptiques", SIAM J. on Control and Optimization 30: 495-521.

[30] Tahraoui, R. (1994) "Maximal torsional rigidity: some qualitative remarks", Proc. Roy. Soc. of Edinburgh 129 A: 971-994.

[31] Turkington, B. (1983) "On steady vortex flow in two dimensions", I-II, Comm. in P.D.E. 9: 999-1071. 
[32] Valadier, M. "Sous-différentiels d'une borne supérieure et d'une somme continue de fonctions convexes", C.R. Acad. Sc. Paris, Série A, 268.

[33] Visintin, A. (1990) "Non-convex functionals related to multiphase systems", SIAM J. Math. Anal. 21: 1281-1304.

[34] Zolesio, J.P. (1981) "The material derivative (or speed) method for shape optimization", in: Optimization of Distributed Parameter Systems, J.Cea \& E. Haug (Eds.), Sijthoff and Noordhoff, Alphen aan den Rijn: 1089-1151.

[35] Zolesio, J.P. (1981) "Domain variational formulation for free boundary problems", in: Optimization of Distributed Parameter Systems, J.Cea \& E. Haug (Eds.), Sijthoff and Noordhoff, Alphen aan den Rijn.

[36] Zolesio, J.P. (1994) "Weak shape formulation for free boundary value problems", Annal. Sc. Norm. Sup. Pisa, Series IV, Vol. XXI: 11-44.

[37] Butazzo, G.; Dal Maso, G. (1993) "An existence result for a class of shape optimization", Arch. Rational Mech. Anal. 122: 183-195. 